\title{
MAGNETO-OPTICAL AND XPS SPECTRA OF COBALT AND TITANIUM SUBSTITUTED HEXAFERRITE FILMS
}

\author{
Zdeněk Šimša, Josef Zemek and Jarmila Šimšová \\ Institute of Physics AS CR, Cukrovarnická 10, 16200 Praha 6, Czech Republic \\ R. Gerber \\ Department of Physics, University of Salford, Salford M5 4WT, UK \\ P. Papakonstantinou, R. Atkinson \\ Department of Pure and Applied Physics, The Queen's University of Belfast, Northern Ireland,UK
}

\begin{abstract}
A combined investigation of the magnetization, Faraday rotation, polar Kerr rotation and the X-ray photoelectron spectroscopy of two Co- and Ti-substituted hexagonal ferrite films, prepared by pulsed laser ablation deposition, was made. The investigation indicates the presence of a certain amount of three valent cobalt ions within the majority of two valent cobalt states. KEYWORDS: HEXAGONAL FERRITES, PHOTOELECTRON SPECTROSCOPY, HYSTERESIS CURVES.
\end{abstract}

\section{INTRODUCTION}

Cobalt substitutions in magnetic garnets, spinels and hexaferrites induce large magneto-optical spectral features that could be exploited in device related applications. The valency states of cobalt ions in substituted hexagonal ferrite films are mostly determined by the other substituent ions. In such a way the four valent titanium is supposed to transform the same concentration of cobalt ions into the two valent state. In analogous garnet systems, however, a simultaneous presence of two and three valent cobalt ions was found even when the concentration of titanium largely exceeded that of cobalt ions. In the paper presented here the combined investigations of the magnetization curves, Faraday rotation (FR), polar Kerr rotation (PKR) and the X-ray photoelectron spectroscopy (XPS) are used in an attempt to determine the valency states of cations in the $\mathrm{Co}$ - and $\mathrm{Ti}$ substituted barium hexaferrites.

\section{EXPERIMENT}

\section{Samples}

The investigations were carried out on two hexagonal ferrite films of the same chemical composition $\mathrm{BaCo}_{0.55} \mathrm{Ti}_{0.60} \mathrm{Fe}_{10.85} \mathrm{O}_{19}$. Films of 90 and $290 \mathrm{~nm}$ thicknesses were prepared by the pulsed laser ablation deposition as described in more detail elsewhere [1]. Both films were highly ordered fine grain polycrystals with their hexagonal c-axis oriented perpendicularly to the (0001) sapphire substrate surface. A slight excess of titanium over the cobalt concentration might lead to the conclusion that all cobalt and at least a part of the iron ions are present in two valent states.

\section{Magnetization}

The in plane and perpendicular hysteresis loops were measured at room temperature either by a VSM or SQUID magnetometer. The results pertaining to the 90 and $290 \mathrm{~nm}$ films are shown in Fig. 1a, b, respectively. They confirme that the magnetic easy axis is directed perpendicularly to the film surface with coercivities of about 1300 and $540 \mathrm{Oe}$ for samples with thicknesses of 90 and $290 \mathrm{~nm}$, respectively. It is further observed that the difference between the magnetic loops in the easy and hard directions is more pronounced for the thicker film.

\section{Magneto-optical properties}

The spectral dependence of the Faraday coefficient was determined for the thicker film by the polarization modulation method as described in [2]. It appears [2-4] that in the near infrared and in a part of the visible spectral region the film is well transparent so that the

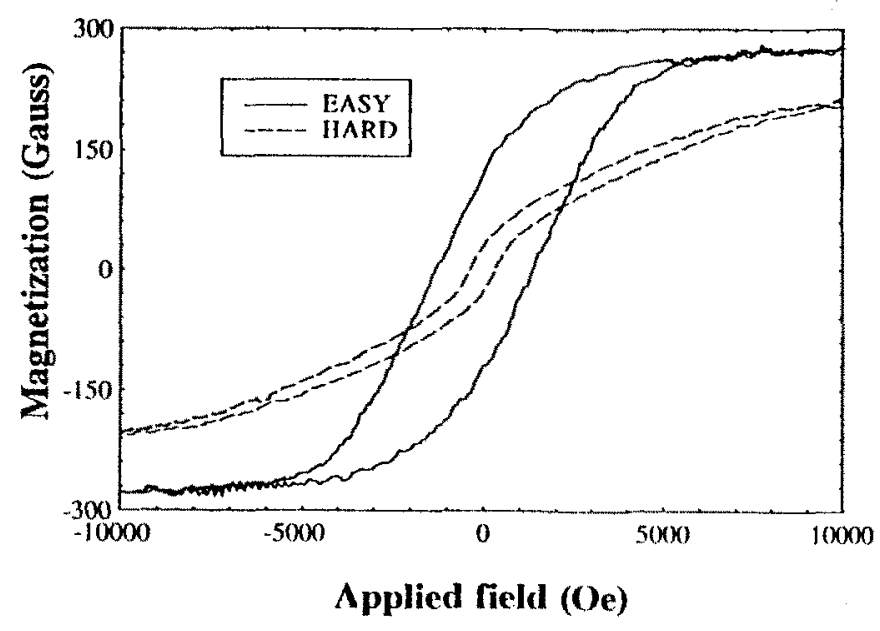

Fig. 1. a) Magnetization hysteresis loops in the easy and hard directions for the $90 \mathrm{~nm}$ thick film. 


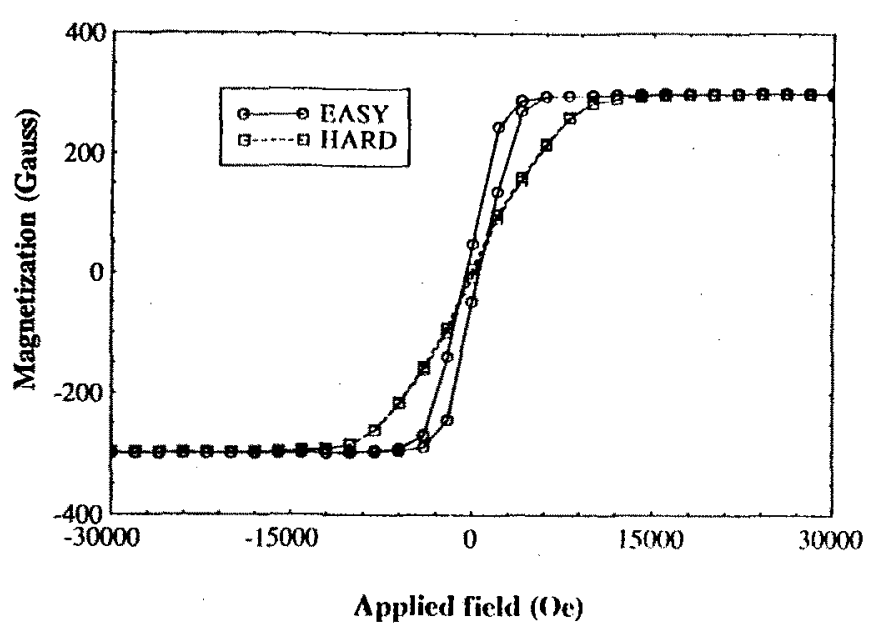

Fig. 1. b) Magnetization hysteresis loops in the easy and hard directions for the $290 \mathrm{~nm}$ thick film.

Faraday rotations could easily be measured. As may be seen from Fig. 2 the largest rotations are found at the photon energies of $0.72,0.85,1.69$ and $2.02 \mathrm{eV}$, corresponding to the wavelengths of $1725,1450,730$ and $610 \mathrm{~nm}$, respectively. These features are mainly due to the presence of cobalt ions as could be inferred from the comparison of this spectra with that of pure barium hexaferrite $(x=0)$, shown in Fig. 2 by the dashed line.

The Faraday rotation hysteresis curves for the largest peaks at 1725 and $1450 \mathrm{~nm}$ for the $290 \mathrm{~nm}$ thick sample are seen in Fig. 3a. The coercivities for both cases are about 550 Oe which is close to the value of 540 Oe obtained by the magnetisation analysis.

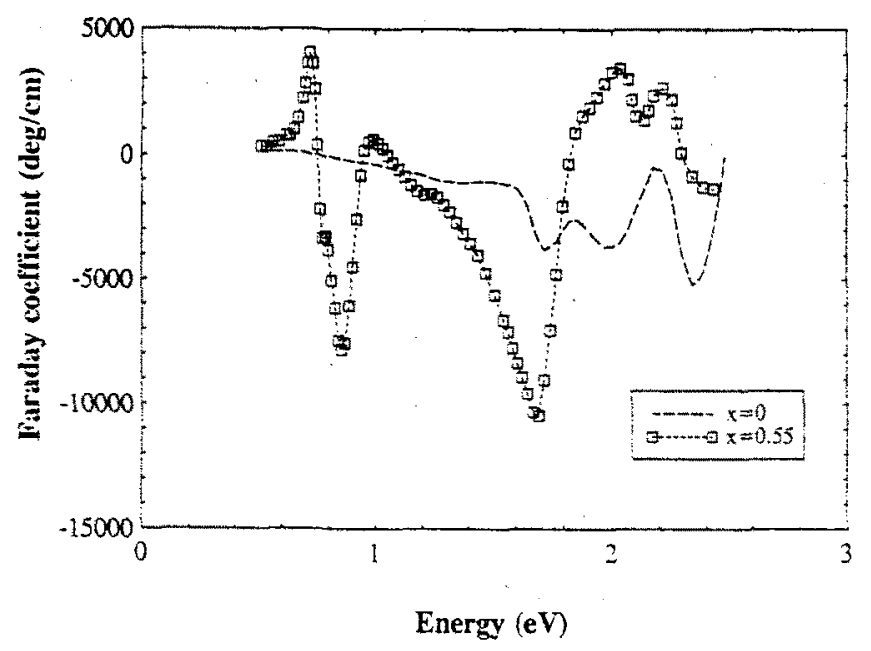

Fig. 2. The spectral dependence of the Faraday coefficient of the $290 \mathrm{~nm}$ thick sample $(x=0.55)$. The dashed line represents the Faraday coefficient of pure barium hexaferrite $(x=0)$.

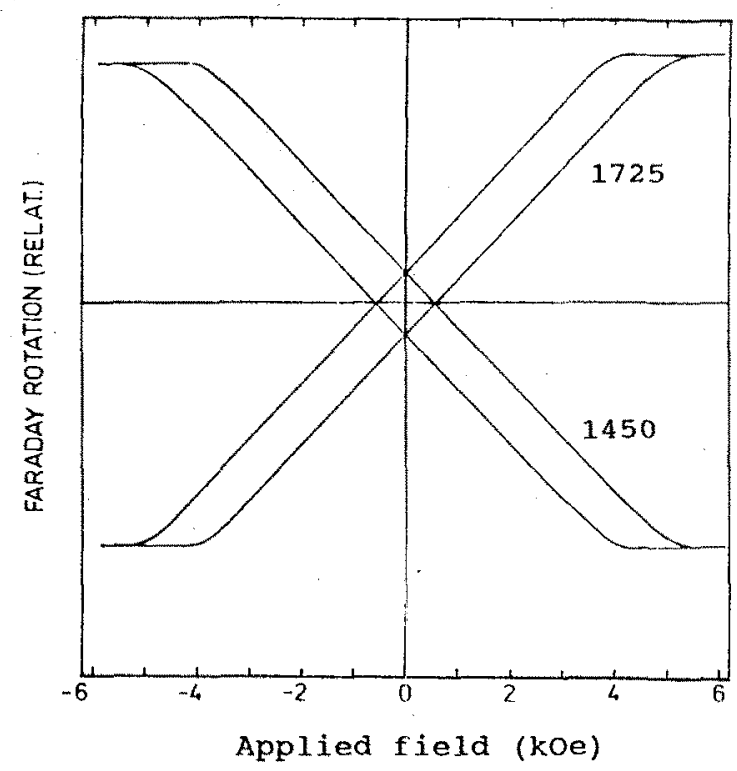

Fig. 3.a) The Faraday rotation hysteresis loops measured at the transparency wavelengths of 1725 and $1450 \mathrm{~nm}$.

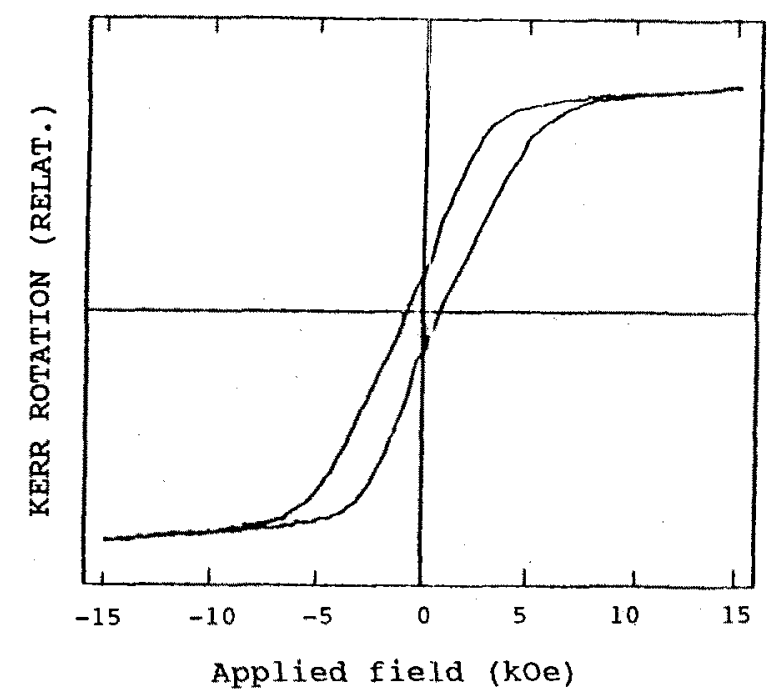

Fig. 3.b) A typical polar Kerr rotation hysteresis loop measured in the nontransparent part of the spectra.

In Fig. 3b, on the other hand, the polar Kerr rotation hysteresis loop is plotted for the wavelengths in the nontransparent part of the spectra, where the Faraday rotation can hardly be measured. As may be seen from Fig. 3.b), the coercive force attains here a value of about 900 Oe.

Hence the magnitude of the "polar Kerr coercivity" as measured in the nontransparent region is higher than that of their magnetic/Faraday rotation counterparts. This is because the Kerr loops give the information only within the skin depth of the probing light beam, while 
the magnetic or Faraday rotation measurements give the information about the sample as a whole. The coercivities obtained from the polar Kerr measurements thus approach those determined from the magnetization measurements on very thin films.

\section{$X P S$}

The spectral dependence of the Faraday coefficient in Fig. 2 shows features characteristic for the presence of the two valent cobalt ions in tetrahedral positions. It was assumed that the $\mathrm{X}$-ray photoelectron spectroscopy could detect the valency of iron and cobalt ions in a similar way as this was the case in magnetic garnets [5]. Therefore, extensive measurements of the XPS spectra were performed on both films using the VGScientific ADES 400 spectrometer and the $\mathrm{Al} \mathrm{K} \mathrm{K}_{\alpha}$ radiation $(1486.6 \mathrm{eV})$. More details about the experimental technique and the method of evaluation of the spectra can be found in [5].

First, it transpired that the position of the main oxygen $\mathrm{O} 1 \mathrm{~s}$ spectral line is very stable with respect to the surface treatment so that its binding energy could be placed at $530.1 \mathrm{eV}$ in agreement with [5]. This placement is also comnensurate with the adsorbed carbon line $\mathrm{C}$ 1s with binding energy of $284.8 \mathrm{eV}$, a value commonly found in magnetic oxides.

The second stable spectrum found was $\mathrm{Ti} 2 \mathrm{p} 3 / 2$ at $458.5 \mathrm{eV}$ and Ti $2 \mathrm{p} 1 / 2$ at $464 \mathrm{eV}$ corresponding to the four valent titanium as is encountered in many other oxides. Sputter-cleaning of the film surface or collecting the photoelectrons at 60 degrees with respect to the surface normal has a negligible effect on these spectra.

The behaviour of the $\mathrm{Fe} 2 \mathrm{p}$ spectra was more variable. The original line positions were found at 711.4 and $724.9 \mathrm{eV}$ for $2 \mathrm{p} 3 / 2$ and $2 \mathrm{p} 1 / 2$ lines, respectively. After sputter cleaning these lines were shifted to 710.6 and $723.8 \mathrm{eV}$ as is demonstrated in Fig. 4. When the spectrum was collected at 60 degrees with respect to the surface normal these values decreased still further to 710.4 and $723.5 \mathrm{eV}$. This behaviour indicates that a transition of a small amount of the original three valent irons to the two valent ions took place.

The Co $2 p$ spectrum, which is found in garnets in the region of 780 to $795 \mathrm{eV}$ and is characteristic for the presence of two and three valent cobalt ions, appeared as the most complicated to interpret. Namely, in barium hexaferrites Co $2 p$ spectrum completely coincides with the more intensive barium $3 \mathrm{~d}$ spectrum as it is demonstrated in Fig. 5. Taking into account the X-ray crosssections of the $\mathrm{Co}$ and $\mathrm{Ba}$ ions one can estimate that the most part of the spectrum in Fig. 5 is due to barium. This is also confirmed by finding that the spectrum

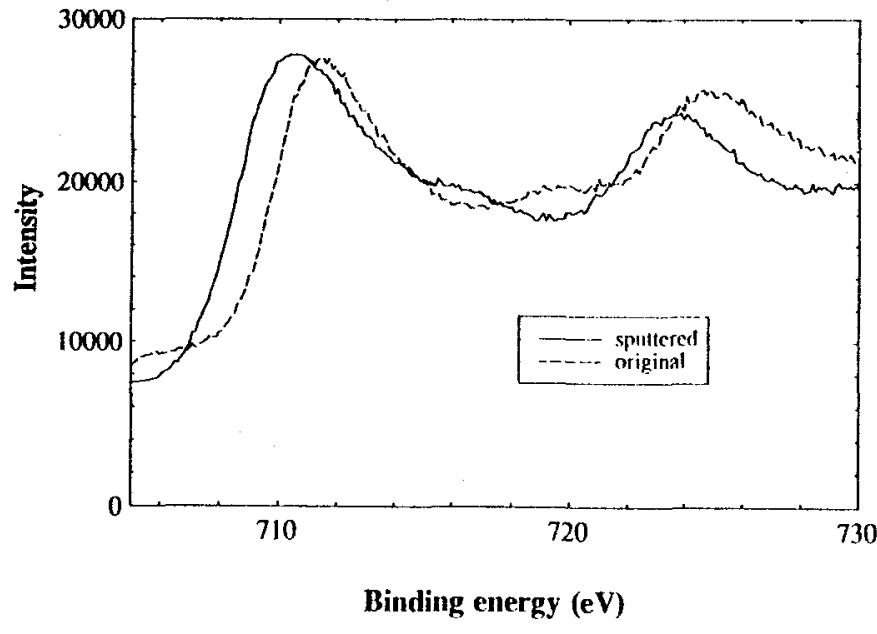

Fig. 4. The XPS spectra in the Fe $2 p$ region for the original and sputter-cleaned $290 \mathrm{~nm}$ film. Sputtercleaning was performed by $\mathrm{Ar}^{+}$accelerated at $3 \mathrm{kV}$ for 3 minutes.

shown has hardly changed by any sputter-cleaning or picking up of photoelectrons at different angles. The second most intensive Co $3 \mathrm{~d}$ region at $60 \mathrm{eV}$ is also of little use for the determination of cobalt valency. Owing to a close proximity of $3 \mathrm{p} 3 / 2$ and $3 \mathrm{p} 1 / 2$ lines any attempt to determine the valency states of cobalt ions from this spectrum appeared to be very difficult.

\section{CONCLUSIONS}

The most direct indication about the valency states of cobalt ions comes from the spectral dependences of the Faraday coefficient. From Fig. 2 and the fitting of the similar curves in bulk hexaferrites [2] it is found

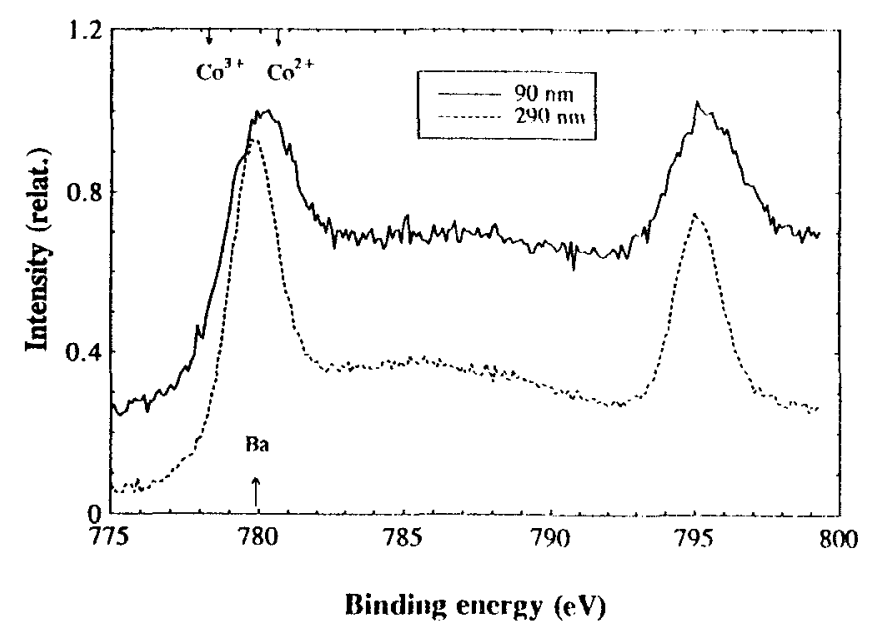

Fig. 5. The XPS spectra of the 90 and $290 \mathrm{~nm}$ films in the Co2p and Ba3d energy region. The arrows show individual ion contributions. 
that the dominant features can be assigned to ${ }^{4} \mathrm{~A}_{2}$ $\rightarrow{ }^{4} \mathrm{~T}_{1}\left({ }^{4} \mathrm{P}\right)$ crystal-field electron transitions of $\mathrm{Co}^{2+}$ in tetrahedral sites. A more detailed discussion of the FR spectra in garnets [6] or thin films of hexaferrites [4] points also to the possibility of the presence of a certain amount of $\mathrm{Co}^{3+}$ together with the two valent cobalt ions in these materials.

Large differences observed by comparing the hysteresis loops in the easy and hard directions for the thin $(90 \mathrm{~nm})$ and thick $(290 \mathrm{~nm})$ films can also be understood in terms of the presence of the cobalt ions, predominantly in the three valent state, on the film surfaces. However, the substantial coercivity of the thinner film can also be explained by the different conditions of preparation leading to the varying surface roughness and/or interfacial stresses arising from the epitaxy. As the film thickness increases the substrate induced stress is partially released resulting in lower coercivities [7].

The most straightforward indication about the valency states of cobalt is usually obtained from the XPS investigation. Unfortunately, in the case of barium hexaferrites this possibility is practically eliminated due to the coincidence of the cobalt and barium spectra. Nevertheless, the detected presence of the two valent iron ions indicates the possibility of the presence of the three valent cobalt as well.

This work has been supported in part by the grant $\# 106 / 96 / 1601$ of the Czech grant agency.

\section{REFERENCES}

[1] P.Papakonstantinou, R. Atkinson, I.W. Salter and R. Gerber, "CoTi- substituted Ba-Ferrite films prepared by pulsed laser deposition", J. Magn. Soc. Japan, 19 S1 (1995) 177.

[2] Z.Šimša, J.Koláček, J.Šimšová, P.Görnert, K.Fischer, R.Gerber, "Faraday rotation in cobaltand titanium-substituted barium hexaferrites", J.Mag.Magn.Mater. 104-107 (1992) 403.

[3] R.Atkinson, R.Gerber, P.Papakonstantinou, I.W.Salter, Z.Šimša, "Optical and magnetooptical properties of $\mathrm{Co} / \mathrm{Ti}$ substituted barium ferrite", J.Mag. Magn.Mater. 104-107 (1992) 1005.

[4] Z.Šimša, R.Gerber, T.Reid, R.Tesař, R.Atkinson and P.Papakonstantinou, "Optical absorption and Faraday rotation of barium hexaferrite films prepared by laser ablation deposition", to be published.

[5] Z.Šimša, J. Zemek, "XPS investigation of LPE garnet films", Czech. J. Phys. 40 (1990) 1274.

[6] Z.Šimša, "Faraday rotation of Co-doped YIG films", IEEE Trans.Magn., MAG-23 (1987) 3323.

[7] P.Papakonstantinou, M. O'Neill, R. Atkinson, I.W. Salter and R. Gerber, "Substrate temperature and oxygen pressure dependence of pulsed laser deposited Sr-ferrite films", J. Magn. Magn. Mater. 152 (1995) 401. 